\title{
A New Method to Track Line Segment in Sequence of Images Using Only its Slope
}

\author{
${ }^{1}$ S. Ait Kaci Azzou and ${ }^{2}$ S. Larabi \\ ${ }^{1}$ Department of Informatique,University of Ferhat Abbas, Setif, Algeria \\ ${ }^{2}$ Department of Informatique, University of Science and Technologie, Houari Boumedienne, Algeria
}

\begin{abstract}
In this paper, we present a novel approach to track a line segment in the sequence of images obtained by camera in motion. We use the slope of segment as feature to track. The proposed method is based only on the formalism of the slope of the segment. We use the definition of the projection of points and the relation which exists between the slopes of the segment in various images. The approach developed not needs the calibration of camera or any knowledge about its movements. To resolve the tracking problem, two segments in three images are necessary. Experiment showed feasibility as well as robustness of the algorithm.
\end{abstract}

Key words: Matching, tracking, estimation of the structure and the motion, projective geometry, computer vision

\section{INTRODUCTION}

Convergence motion of cameras, similar to the movements of eyes, constitutes a subject of search in artificial vision. The introduction in the algorithms of stereoscopic vision allows to get closer to the human perception which is essentially dynamic ${ }^{[1]}$.

Indeed the estimation of the movement is important for various applications such as: 3D reconstruction, objects tracking and the visual subjection.

Various methods were developed for the estimation of the movement and the structure ${ }^{[2,3]}$. We distinguish two classes of methods; the first is based on the calculation of the optical flow and the spatio-temporal relations ${ }^{[4]}$; the second's on the feature matching ${ }^{[5,6]}$.

In this last case, used approaches are based generally on Euclidian's models and require a calibration of cameras ${ }^{[7-9]}$; uses the matching to resolve the problem of the movement and the structure; but there are practically no methods which use movement to resolve the problem of the feature tracking.

Our goal is to resolve the feature tracking problem in a sequence of images obtained by camera in rotation motion, without any knowledge about geometrical models of camera or of its movements.

Our method is based only on the formalism of the slope of the segment, using projective coordinates of extremities of the segment in the image plane.

In this article, we present at first the theoretical aspects and the algorithmic of a tracking method, based on the calculation of the slopes of a line segment in the projective space. We also present the experimental results obtained by tested algorithm on different images.

\section{POSITION OF THE PROBLEM}

\section{Hypotheses}

* We have a binocular system of vision not anthropomorphic in convergence motion;

* We suppose that every image was segmented and the contour approximated by line segments.

* Let us note $\mathrm{IM}_{0}, \mathrm{IM}_{1}, \mathrm{IM}_{2}, . ., \mathrm{IM}_{\mathrm{I}}, \ldots, \mathrm{IM}_{\mathrm{N}}$ the sequence of images taken with the camera where $\mathrm{IM}_{\mathrm{I}}$ is image obtained with the camera after I rotations.

* $\quad \mathrm{IM}_{\mathrm{I}}=\left\{\mathrm{S}_{1, \mathrm{i}}, \mathrm{S}_{2, \mathrm{i}}, \mathrm{S}_{3, \mathrm{I}}, \ldots, \mathrm{S}_{\mathrm{k}, \mathrm{I}}, \ldots \mathrm{S}_{\mathrm{n}, \mathrm{I}}\right\}$ where $\mathrm{S}_{\mathrm{kI}}$ is the segment $\mathrm{k}$ in the image I obtained by the camera after the I rotations.

* Every segment is defined with its two point's extremities.

Geometrical model of convergence motion: The camera is placed on a support plan and when it makes a movement of rotation, the centre of projection likened to a point is considered as moving on the same plan.

Because of the uncertainties of the mechanics, we take place in the unfavourable case where the centre of rotation of the camera is not unique (the theoretical optical axes intersect in various points) to see Fig. 1. This has no repercussion on the proposed algorithm because it is independent from the centre of rotation. The geometrical model of the movement of rotation of the camera is illustrated by it Fig. 2.

Where

* IM is projective plan

* $\mathrm{P}$ is the intersection of the optical axis with the image plan

* PUV is the theoretical frame in IM

* $\quad \mathrm{L}$ is the centre of projection

* $\quad \mathrm{PL}=\mathrm{f}$ (focal length) 

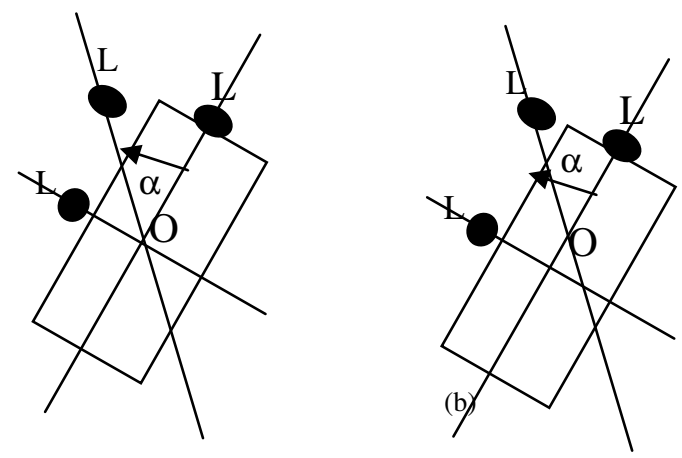

Fig. 1: Example of camera in rotation motion, (a) With fixed rotation centre (b) variable centre of rotation

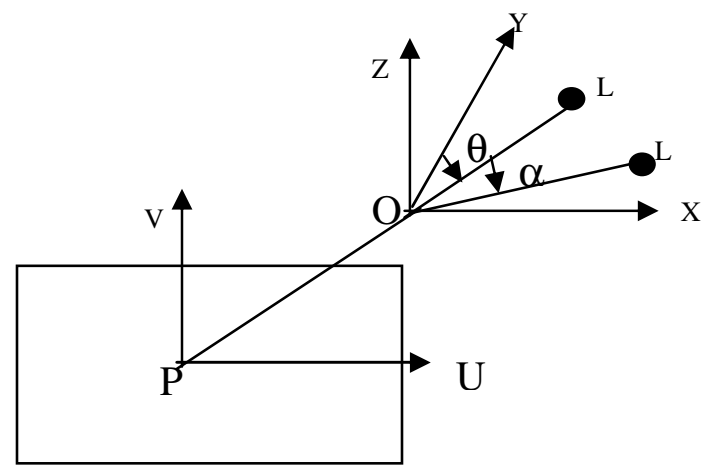

Fig. 2: Geometrical model of camera's motion

* $\theta$ is the initial angle between the optical axes PL and $\mathrm{OY}$

* $\quad \alpha$ is the angle of rotation of the camera

* $\mathrm{O}$ is the theoretical centre of the rotation of the camera, obtained as intersection of the optical axes corresponding to the two states of the camera

* $\quad \mathrm{PO}=\mathrm{d}$

* (OXYZ) is a external orthonormal frame of the camera; it can be fixed such as OX //PU, OY coincides with the optical axis and $\mathrm{OZ} / / \mathrm{PV}$

* ex, ez define the dimensions of the pixel

In our theoretical study none of these parameters are supposed known.

For any point $\mathrm{M}(\mathrm{x}, \mathrm{y}, \mathrm{z})$ of the $3 \mathrm{D}$ space, it projective coordinates $\mathrm{u}_{\mathrm{M}}, \mathrm{v}_{\mathrm{M}}$ on IM are $\mathrm{a}^{[10]}$ :

$u_{M}=e x \cdot f \cdot \frac{-\operatorname{Cos}(\theta) \cdot x+\sin (\theta) \cdot y}{\sin (\theta) \cdot x+\cos (\theta) \cdot y+d-f} ;$

$v_{M}=e z \cdot f \cdot \frac{-z}{\sin (\theta) \cdot x+\cos (\theta) \cdot y+d-f}$

After rotation motion of $\alpha$, the new projective coordinates of $\mathrm{M}$ become

$u_{M}=e x \cdot f \cdot \frac{-\operatorname{Cos}(\theta+\alpha) \cdot x+\sin (\theta+\alpha) \cdot y}{\sin (\theta+\alpha) \cdot x+\cos (\theta+\alpha) \cdot y+d-f} ;$

$v_{M}=e z \cdot f \cdot \frac{-z}{\sin (\theta+\alpha) \cdot x+\cos (\theta+\alpha) \cdot y+d-f}$
We have only projective coordinates of line segment's extremities in images sequences and their mathematical formulations.

The method of line segment matching is based only on the formalism of projective point's extremities of segments.

\section{MATERIALS AND METHODS}

Choice of the primitive: We use in this method the slope of the segment as feature tracking.

This choice is justified by the fact that the slope of the segment is robust to the noise and also it is independent from the used frame ${ }^{[11]}$.

Theoretical Aspects of the method: - $S_{i, 0}$ line segment $\mathrm{i}$ of $\mathrm{IM}_{0}$.

* $S_{i, 1}$ line segment $i$ of $\mathrm{IM}_{1}$ obtained after rotation of an angle $\alpha$ of the camera.

Let us note $\mathrm{v}_{0}=\mathrm{a}_{\mathrm{i} 0}$. $\mathrm{U}_{0}+\mathrm{b}_{\mathrm{i}, 0}$ the equation of $\mathrm{S}_{\mathrm{i}, 0}$ with regard to the frame (PUV).

Projective coordinates on $\mathrm{IM}_{0}$ of every point $\mathrm{Mi}(\mathrm{xi}$, yi, zi) of the scene $3 \mathrm{D}$ are:

$u_{0}=e x . f \cdot \frac{-X_{i, 0}}{Y_{i, 0}+d-f} ; v_{0}=e z \cdot f \cdot \frac{-Z_{i, 0}}{Y_{i, 0}+d-f}$

$\left\{\begin{array}{l}X_{i, 0}=\mathrm{X}_{\mathrm{i}} \cdot \cos (\theta)-y_{i} \cdot \sin (\theta) \\ Y_{i, 0}=\mathrm{X}_{\mathrm{i}} \cdot \sin (\theta)+y_{i} \cdot \cos (\theta) \\ Z_{i, 0}=\mathrm{Z}_{\mathrm{i}} \cdot\end{array}\right.$

Equation $\mathrm{v}_{0}=\mathrm{a}_{\mathrm{i}, 0} \cdot \mathrm{U}_{0}+\mathrm{b}_{\mathrm{i}, 0}$ of the segment $\mathrm{S}_{\mathrm{i}, 0}$ become:

$$
z_{i, 0}=a_{i, 0} \frac{e x}{e z} \cdot X_{i, 0}-\frac{b_{i, 0}}{e z . f} \cdot\left(Y_{i, 0}+d-f\right)
$$

In the same way, equation $\mathrm{v}_{1}=\mathrm{a}_{\mathrm{i}, 1} \cdot \mathrm{u}_{1}+\mathrm{b}_{\mathrm{i}, 1}$ of the segment $S_{i, 1}$ (homologue of $S_{i, 0}$ after camera's rotation of an angle $\alpha$ ) is as follows:

$$
z_{i, 1}=a_{i, 1} \cdot \frac{e x}{e z} \cdot X_{i, 1}-\frac{b_{i, 1}}{e z \cdot f} \cdot\left(Y_{i, 1}+d-f\right)
$$

where

$$
\left\{\begin{array}{l}
X_{i, 1}=\mathrm{X}_{\mathrm{i}} \cdot \cos (\theta+\alpha)-y_{i} \sin (\theta+\alpha)=\mathrm{X}_{\mathrm{i}, 0^{\circ}} \cdot \cos (\alpha)-Y_{i, 0} \cdot \sin (\alpha) \\
Y_{i, 1}=\mathrm{X}_{\mathrm{i}} \cdot \sin (\theta+\alpha)+y_{i} \cdot \cos (\theta+\alpha)=\mathrm{X}_{\mathrm{i}, 0} \cdot \sin (\alpha)+Y_{i, 0^{\prime}} \cdot \cos (\alpha) \\
Z_{i, 1}=\mathrm{Z}_{\mathrm{i}}=Z_{i, 0}
\end{array}\right.
$$

Expressing $X_{i, 0}$ and $Y_{i, 0}$ according to $X_{i, 1}$ and $Y_{i, 1}$ :

$$
\begin{aligned}
Z_{i, 1} & =X_{i, 1}\left[\frac{e x}{e z} a_{i, 0} \cdot \cos (\alpha)+\frac{b_{i, 0}}{f . e z} \cdot \sin (\alpha)\right] \\
& -\frac{Y_{i, 1}}{f . e x}\left[f \cdot e x \cdot a_{i, 0} \cdot \sin (\alpha)-b_{i, 0} \cdot \cos (\alpha)\right] \\
& -\frac{b_{i, 0}}{f . e z}[d-f]
\end{aligned}
$$


Using the equations (2) and (3), we obtain:

$\frac{e x}{e z} a_{i, 1}=\frac{1}{\text { f.ez }}\left[f \cdot e x a_{i, 0} \cdot \cos (\alpha)+b_{i, 0} \cdot \sin (\alpha)\right]$

where

$\frac{a_{i, 1}-a_{i, 0} \cdot \cos (\alpha)}{\sin (\alpha)}=\frac{b_{i, 0}}{f . e x}$

In the same way, by considering the segment $S_{i, 2}$ (homologue of $\mathrm{S}_{\mathrm{i}, 0}$ after camera's rotation of an angle $\beta)$ expression (4) become :

$\frac{a_{i, 2}-a_{i, 0} \cdot \cos (\beta)}{\sin (\beta)}=\frac{b_{i, 0}}{f . e x}$

For all three images of sequence of the same line segment, the follows expression is only defined with slopes of segment and rotation motion of camera:

$a_{i, 1} \cdot \sin (\beta)-a_{i, 2} \cdot \sin (\alpha)=a_{i, 0} \cdot \sin (\beta-\alpha)$

In the same way, by considering the segment $S_{j}$ in the same sequence of images we obtain:

$a_{j, 1} \cdot \sin (\beta)-a_{j, 2} \cdot \sin (\alpha)=a_{j, 0} \cdot \sin (\beta-\alpha)$

The report (6) / (7) lead us to the following formula:

$\frac{a_{i, 1} \cdot \sin (\beta)-a_{i, 2^{2}} \sin (\alpha)}{a_{j, 1} \cdot \sin (\beta)-a_{j, 2} \cdot \sin (\alpha)}=\frac{a_{i, 0} \cdot \sin (\beta-\alpha)}{a_{j, 0} \cdot \sin (\beta-\alpha)}=\frac{a_{i, 0}}{a_{j, 0}}$.

We can write (e) us:

$\frac{a_{i, 1} \cdot a_{j, 0}-a_{i, 0} \cdot a_{j, 1}}{a_{j, 0} \cdot a_{i, 2}-a_{i, 0} \cdot a_{j, 2}}=\frac{\sin (\alpha)}{\sin (\beta)} \cdot=C_{012}$

Note that $C_{012}$ is a constant witch is function of the two motions ( $\alpha$ and $\beta$ ), done by the camera.

This constant is estimated by using the slopes of two line segments in three images of sequence.

For all segment considered in the sequence of images $\mathrm{IM}_{0}, \mathrm{IM}_{1}$ and $\mathrm{IM}_{2}$.

Note, $\mathrm{R}$ the function that calculates this constant.

$\mathrm{R}$ is defined as follows:

$\mathrm{R}: \mathrm{IM}_{0} \mathrm{xIM}_{0} \mathrm{xIM}_{1} \mathrm{xIM} \mathrm{IM}_{1} \mathrm{IM}_{2} \mathrm{xIM}_{2} \rightarrow \mathrm{R}$

$\mathrm{R}\left(\mathrm{S}_{\mathrm{i}, 0}, \mathrm{~S}_{\mathrm{j}, 0}, \mathrm{~S}_{\mathrm{i}, 1}, \mathrm{~S}_{\mathrm{j}, 1}, \mathrm{~S}_{\mathrm{i}, 2}, \mathrm{~S}_{\mathrm{j}, 2}\right)=C_{012}$

For all three segment $\left(\mathrm{S}_{\mathrm{i}}, \mathrm{S}_{\mathrm{j}}, \mathrm{S}_{\mathrm{k}}\right) \mathrm{C}_{012}$ is estimated at the same manner.

We can then express the following proposition:

Proposition: $\quad\left(\mathrm{S}_{\mathrm{i}, 0}, \mathrm{~S}_{\mathrm{j}, 0}, \mathrm{~S}_{\mathrm{k}, 0}\right), \quad\left(\mathrm{S}_{\mathrm{i}, 1}, \mathrm{~S}_{\mathrm{j}, 1}, \mathrm{~S}_{\mathrm{k}, 1}\right) \quad$ and $\left(\mathrm{S}_{\mathrm{i}, 2}, \mathrm{~S}_{\mathrm{j}, 2}, \mathrm{~S}_{\mathrm{k}, 2}\right)$ three triplets of line segments in three images $\mathrm{IM}_{0}, \mathrm{IM}_{1}$ and $\mathrm{IM}_{2}$ (Fig. 3). The necessary condition to match this three triplets of line segment is: $\mathrm{R}\left(\mathrm{S}_{\mathrm{i}, 0}, \mathrm{~S}_{\mathrm{j}, 0}, \mathrm{~S}_{\mathrm{i}, 1}, \mathrm{~S}_{\mathrm{j}, 1}, \mathrm{~S}_{\mathrm{i}, 2}, \mathrm{~S}_{\mathrm{j}, 2}\right)=\mathrm{R}\left(\mathrm{S}_{\mathrm{i}, 0}, \mathrm{~S}_{\mathrm{k}, 0}, \mathrm{~S}_{\mathrm{i}, 1}, \mathrm{~S}_{\mathrm{k}, 1}, \mathrm{~S}_{\mathrm{i}, 2}\right.$, $\left.\mathrm{S}_{\mathrm{k}, 2}\right)=\mathrm{R}\left(\mathrm{S}_{\mathrm{j}, 0}, \mathrm{~S}_{\mathrm{k}, 0}, \mathrm{~S}_{\mathrm{j}, 1}, \mathrm{~S}_{\mathrm{k}, 1}, \mathrm{~S}_{\mathrm{j}, 2}, \mathrm{~S}_{\mathrm{k}, 2}\right)$

The solution we propose has a way similar to that current in Hough's transformed ${ }^{[12]}$ : it is a question of engendering all the possibilities for the correspondence constant $C_{012}$ and keeping that collect the maximum of votes.
$C_{012}$ Represent the matching factor.

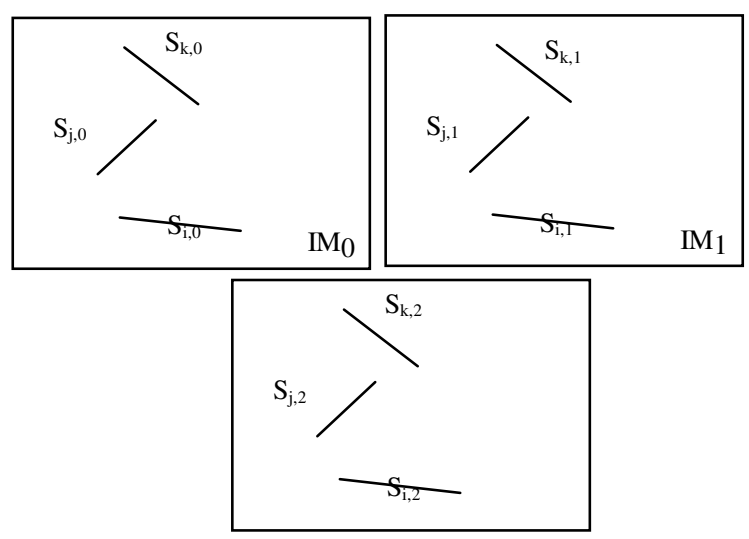

Fig. 3: Exemple of 3 line segments in 3 images

\section{Tracking algorithm}

For all pair of segment $\left(\mathrm{S}_{\mathrm{i}, 0}, \mathrm{~S}_{\mathrm{j}, 0}\right)$ of $\mathrm{IM}_{0}$

Do For all $\left(\mathrm{S}_{\mathrm{i}, 1}, \mathrm{~S}_{\mathrm{j}, 1}\right)$ of $\mathrm{IM}_{1}$ Do for all $\left(\mathrm{S}_{\mathrm{i}, 2}, \mathrm{~S}_{\mathrm{j}, 2}\right)$ of $\mathrm{IM}_{2}$

Do Estimate $\mathrm{R}\left(\mathrm{S}_{\mathrm{i}, 0}, \mathrm{~S}_{\mathrm{j}, 0}, \mathrm{~S}_{\mathrm{i}, 1}, \mathrm{~S}_{\mathrm{j}, 1}, \mathrm{~S}_{\mathrm{i}, 2}, \mathrm{~S}_{\mathrm{j}, 2}\right)=C$ end ;

end;

end;

Search the value of $C$ witch is engendered by the maximum of segments.

Note $C_{012}$ this value.

All the triplets of pair of line segments witch engendered $C_{012}$ are in correspondences.

end.

\section{RESULTS}

The tracking algorithm tested on synthetically data generated by computer gives expected results on noisy and not noisy data. Applied also to real images segmented by Canny-Deriche's method, the proposed algorithm gives suitable results (Fig. 4).

In this method, we remind that:

* The extremities of segments are indicated manually.

* The vertical segments are eliminated; then to be matched by using the constraints of order and neighbourhood.

Furthermore, experiment shows that algorithm gives better results for the oblique segments $\left(<=45^{\circ}\right)$ and for the long segments with regard to the short segments.

\section{CONCLUSION}

We have proposed a method of tracking line segments by using as primitive the slope of the segment 
which is a robust feature. This method does not require any knowledge of the geometrical models of the camera or of its motion. The experimental results showed that the algorithm is robust until three pixels of noises and it gives better results for the oblique segments $\left(<=45^{\circ}\right)$ and for the long segments with regard to the short segments. This tracking process supplied as result the trajectory of the primitives which are going to be used to resolve a problem of stereoscopic matching.

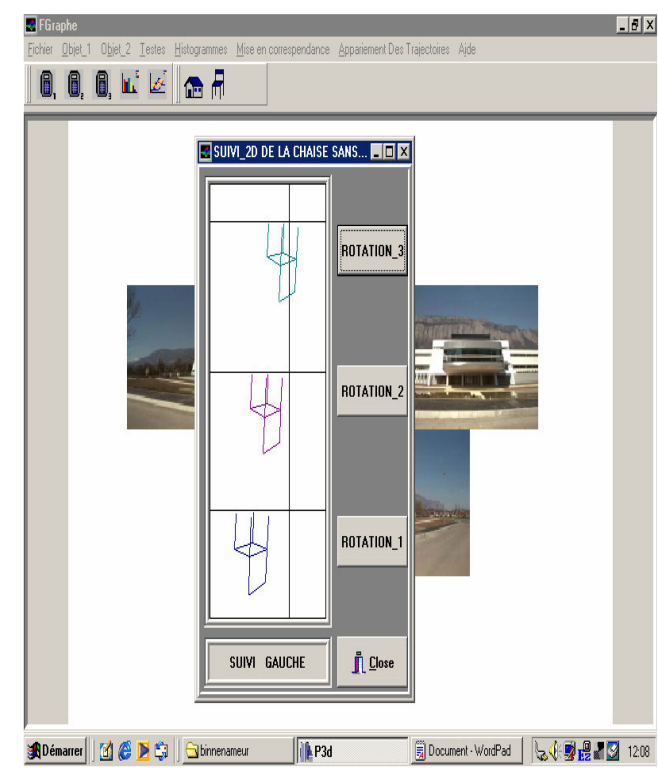

After suppression of vertical segments and revolving systems the matching segments are as follow. The vertical segments are matched using neighborhood constraints.

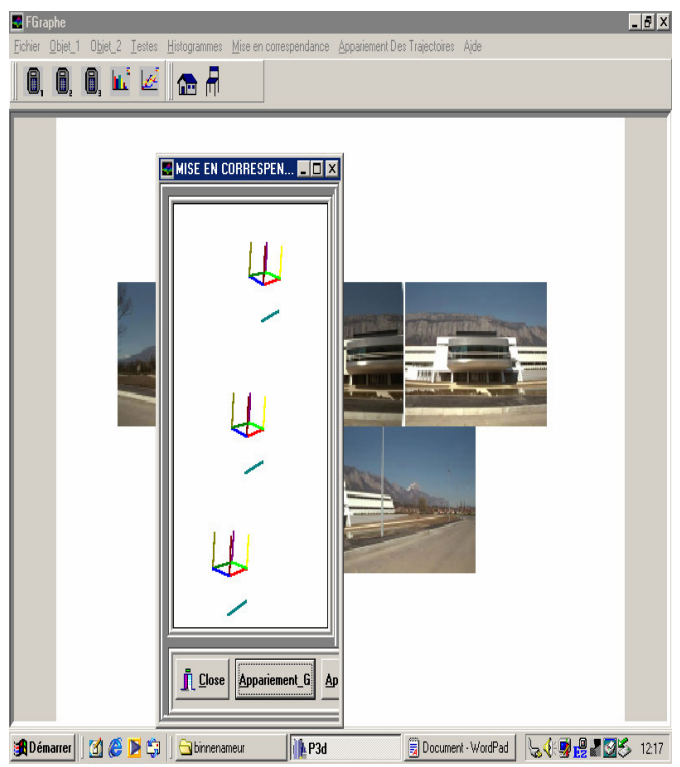

Fig. 4: Example of object in the sequence of 3 images

\section{REFERENCES}

1. Faugeras, O.D, 1988. Quelques pas vers la vision artificielle en trois dimensions. TSI Techniques et Sciences Informatiques, 7: 7.

2. Bazin, P.L. and J.M. Vezien, 2000. Tracking geometric primitives in video streams. Proc. 4th Irish Machine Vision and Image Processing Conf., pp: 43-50. Balfast.

3. Martine, D. and T. Pajdla, 2003. Line reconstruction from many perspective images by factorization. Proc. Conf. Computer Vision and Pattern Recognition, Madison, Wisconsin, USA, 1: 497-502, IEEE Computer Society Press.

4. Dekeyser, F., P. Bouthemy, P. Perez and E. Payot, 2000. Super-resolution from noisy image sequence exploiting a 2D parametric model. LAPRInt, Conf. Pattern Recognition, 3: 354-357, Barcelone, ESP.

5. Bartoli, A. and P. Sturm, 2003. Multiple view structure and motion from line correspondences. Extended version -Short version appeard in the 9th ICCV Nice, France.

6. Huang, T.S. and A.N. Netravali, 1994. Motion and structure from feature correspondences: A review. Proc. IEEE, 82: 252-267.

7. Faugeras, O. and B. Mourrain, 1996. On the geometry and algebra of the points and line correspondances between $\mathrm{n}$ images. Proc. 5th Intl. Conf. Computer Vision, Cambridge Massachussetts,USA, pp: 951-956.

8. Meer, P., D. Mintz, D.Y. Kim and Rosenfeld, 1991. A robust regression methods in computer vision: A review. Intl. J. Computer Vision, 6 :5970 .

9. Spetsakis, M. and J. Aloimonos, 1990. Structure from motion using linecorrespondances. Intl. J. Computer Vision, 4: 171-1.

10. Duda, R. and P. Hart, 1973. Pattern Classification and Scene Analysis. Wiley Interscience Publication.

11. Azzou, S.A.K. and S. Larabi, 1996. Appariement de trajectoires issue d'un système de vision stéréoscopique en mouvement de convergence.ICAOS 96, 15 ème Conférence Internationale du traitement d'images Paris .

12. Hough, 1962. Method and means for recognizing complex patterns. U.S. Patent 3069654. 